\title{
Solutions of the Dirac Equation in a Magnetic Field and Intertwining Operators ${ }^{\star}$
}

\author{
Alonso CONTRERAS-ASTORGA ${ }^{\dagger}$, David J. FERNÁNDEZ C. ${ }^{\dagger}$ and Javier NEGRO ${ }^{\ddagger}$ \\ $\dagger$ Departamento de Física, Cinvestav, AP 14-740, 07000 México DF, Mexico \\ E-mail: acontreras@fis.cinvestav.mx,david@fis.cinvestav.mx \\ ¥ Departamento de Física Teórica, Atómica y Óptica, Universidad de Valladolid, \\ 47071 Valladolid, Spain \\ E-mail: jnegro@fta.uva.es
}

Received July 31, 2012, in final form October 17, 2012; Published online October 28, 2012

http://dx.doi.org/10.3842/SIGMA.2012.082

\begin{abstract}
The intertwining technique has been widely used to study the Schrödinger equation and to generate new Hamiltonians with known spectra. This technique can be adapted to find the bound states of certain Dirac Hamiltonians. In this paper the system to be solved is a relativistic particle placed in a magnetic field with cylindrical symmetry whose intensity decreases as the distance to the symmetry axis grows and its field lines are parallel to the $x-y$ plane. It will be shown that the Hamiltonian under study turns out to be shape invariant.
\end{abstract}

Key words: intertwining technique; supersymmetric quantum mechanics; Dirac equation

2010 Mathematics Subject Classification: 81Q05; 81Q60; 81Q80

\section{Introduction}

The intertwining technique, also called Supersymmetric Quantum Mechanics (SUSY QM), is a widespread method used to generate exactly solvable Hamiltonians departing from a given initial one and can be employed as well to solve a certain set of Hamiltonians in a closed way, among other applications. In the simplest case (1-SUSY QM) the new potentials have similar spectra as the original one, namely, they might differ at most in the ground state energy. Examples of potentials generated by this technique are those which arise when adding a bound state to the free particle Hamiltonian (hyperbolic Pöschl-Teller) [14] or the Abraham-MosesMielnik potentials which are isospectral to the harmonic oscillator [1, 13, 15]. This method has been also applied successfully to the radial part of the hydrogen atom potential $[1,7,13,18]$, the trigonometric Pöschl-Teller potentials [3], among many others.

To apply the technique [8] we start from two one-dimensional Schrödinger Hamiltonians

$$
H_{i}=-\frac{1}{2} \frac{\mathrm{d}^{2}}{\mathrm{~d} x^{2}}+V_{i}(x), \quad i=0,1,
$$

where $H_{0}$ is known. Now let us suppose the existence of a differential operator $A_{1}^{\dagger}$ which satisfies

$$
H_{1} A_{1}^{\dagger}=A_{1}^{\dagger} H_{0}, \quad A_{1}^{\dagger}=\frac{1}{\sqrt{2}}\left(-\frac{\mathrm{d}}{\mathrm{d} x}+W_{1}(x)\right) .
$$

Since the operator $A_{1}^{\dagger}$ is of first order, the technique is known as 1-SUSY QM and the function $W_{1}(x)$ as the superpotential. It is also said that the potentials $V_{0}(x)$ and $V_{1}(x)$, whose Hamiltonians are intertwined by the operator $A_{1}^{\dagger}$, are supersymmetric partners.

\footnotetext{
*This paper is a contribution to the Special Issue "Superintegrability, Exact Solvability, and Special Functions". The full collection is available at http://www.emis.de/journals/SIGMA/SESSF2012.html
} 
In order to satisfy equation $(1) V_{1}(x)$ and $W_{1}(x)$ must obey

$$
V_{1}(x)=V_{0}(x)-W_{1}^{\prime}(x), \quad W_{1}^{\prime}(x)+W_{1}^{2}(x)=2\left(V_{0}-\epsilon_{1}\right),
$$

where $\epsilon_{1}$ is a real integration constant called factorization energy. From the previous equations we can see that if $V_{0}(x)$ is given and $W_{1}(x)$ is found, the supersymmetric partner $V_{1}(x)$ is completely determined. Furthermore, equation (1) ensures that if $\psi_{n}$ is an eigenfunction of $H_{0}$ with eigenvalue $E_{n}$ then $A_{1}^{\dagger} \psi_{n}$ will be an eigenfunction of $H_{1}$ with the same eigenvalue. Note that the operators $A_{1}^{\dagger}$ and $\left(A_{1}^{\dagger}\right)^{\dagger} \equiv A_{1}$ factorize the Hamiltonian as follows

$$
H_{0}=A_{1} A_{1}^{\dagger}+\epsilon_{1}, \quad H_{1}=A_{1}^{\dagger} A_{1}+\epsilon_{1},
$$

where

$$
A_{1}=\frac{1}{\sqrt{2}}\left(\frac{\mathrm{d}}{\mathrm{d} x}+W_{1}(x)\right) .
$$

By taking the squared norm of the vectors $A_{1}^{\dagger} \psi_{n}$ we have $\left\|A_{1}^{\dagger} \psi_{n}\right\|^{2}=\left\langle A_{1}^{\dagger} \psi_{n}, A_{1}^{\dagger} \psi_{n}\right\rangle=$ $\left\langle\psi_{n}, A_{1} A_{1}^{\dagger} \psi_{n}\right\rangle=E_{n}-\epsilon_{1} \geq 0 \forall n$ which implies that $\epsilon_{1} \leq E_{0}$, where $E_{0}$ is the ground state energy of $H_{0}$. One could ask now if $\left\{A_{1}^{\dagger} \psi_{n}, n=0,1,2, \ldots\right\}$ is a complete orthogonal set. In order to answer this question, assume the existence of a vector $\psi_{\epsilon_{1}}$ orthogonal to each vector of the previous set, then

$$
\left\langle\psi_{\epsilon_{1}}, A_{1}^{\dagger} \psi_{n}\right\rangle=\left\langle A_{1} \psi_{\epsilon_{1}}, \psi_{n}\right\rangle=0 \quad \forall n \quad \Rightarrow \quad A_{1} \psi_{\epsilon_{1}}=0,
$$

since $\left\{\psi_{n}, n=0,1,2, \ldots\right\}$ is a complete orthogonal set. The first-order differential equation $A_{1} \psi_{\epsilon_{1}}=0$ can be solved immediately

$$
\psi_{\epsilon_{1}} \propto \exp \left[-\int_{0}^{x} W_{1}(y) \mathrm{d} y\right] .
$$

Notice that $\psi_{\epsilon_{1}}$ satisfies

$$
H_{1} \psi_{\epsilon_{1}}=\epsilon_{1} \psi_{\epsilon_{1}}
$$

Thus, depending on the square integrability of this vector, and the value of $\epsilon_{1}$, three possibilities arise

- The function $\psi_{\epsilon_{1}}$ with $\epsilon_{1}<E_{0}$ belongs to the Hilbert space $\mathcal{H}$. Thus, $\left\{\psi_{\epsilon_{1}}, A_{1}^{\dagger} \psi_{n}, n=\right.$ $0,1,2, \ldots\}$ is a complete orthogonal set, and from equations (1), (3) the spectrum of $H_{1}$ is given by $\operatorname{Sp}\left[H_{1}\right]=\left\{\epsilon_{1}, E_{n}, n=0,1,2, \ldots\right\}$.

- $\psi_{\epsilon_{1}} \notin \mathcal{H}$ with $\epsilon_{1}<E_{1}$. In this case $\left\{A_{1}^{\dagger} \psi_{n}, n=0,1,2, \ldots\right\}$ is a complete orthogonal set and thus $\operatorname{Sp}\left[H_{1}\right]=\operatorname{Sp}\left[H_{0}\right]$.

- When $\psi_{\epsilon_{1}} \notin \mathcal{H}, \epsilon_{1}=E_{0}$, the set $\left\{A_{1}^{\dagger} \psi_{n}, n=1,2,3, \ldots\right\}$ is complete and thus $\operatorname{Sp}\left[H_{1}\right]=$ $\left\{E_{n}, n=1,2,3, \ldots\right\}$.

Restricting ourselves to this last case, it can be verified that $W_{1}(x)=\psi_{0}^{\prime} / \psi_{0}$ fulfills equation (2) and applying successively this technique we can generate a hierarchy of Hamiltonians, where $\operatorname{Sp}\left[H_{0}\right] \supset \mathrm{Sp}\left[H_{1}\right] \supset \mathrm{Sp}\left[H_{2}\right] \supset \cdots$. This sequence is either finite or infinite if the number of bound states of $H_{0}$ is finite or infinite respectively [21].

Up to this point we have assumed that starting from a solvable Hamiltonian $H_{0}$ we can generate a hierarchy of Hamiltonians $\left\{H_{i}, i=0,1, \ldots\right\}$. However, from the equation adjoint to equation (1) we can see that beginning from an eigenvector of $H_{i}$ we can construct an eigenvector 
of $H_{i-1}$ through the action of the operator $A_{i}$. Thus, if we had known enough eigenvectors of the Hamiltonians of the hierarchy, for example all the ground states, it would be possible to build all bound states of $H_{0}$ by applying the operators $A_{i}$ over them.

It is convenient to recall now the concept of shape invariance. If two SUSY partner potentials $V_{1,2}\left(x ; a_{1}\right)$ satisfy the condition

$$
V_{2}\left(x ; a_{1}\right)=V_{1}\left(x ; a_{2}\right)+R\left(a_{1}\right),
$$

where $a_{1}$ is a set of parameters, $a_{2}$ is a function of $a_{1}$ and the remainder $R\left(a_{1}\right)$ is independent of $x$, then $V_{1}\left(x ; a_{1}\right)$ and $V_{2}\left(x ; a_{1}\right)$ are said to be shape invariant [4].

If the potentials of a hierarchy of Hamiltonians are shape invariant and the ground state of one of them is found, in principle, all the ground states can be derived. In this way we can find the eigenfunctions of the first Hamiltonian. The harmonic oscillator and the radial effective potential of the hydrogen atom are examples of shape invariant potentials that can be solved through this procedure.

It is noteworthy that there are papers in which through the SUSY technique the Dirac equation for different systems has been solved [2, 5, 6, 12, 16, 17, 20] or analyzed [11]. However, the differences with respect to the approach we will use here will be significant.

In Section 2 we will employ the 1-SUSY QM in order to solve the stationary Schrödinger equation for a charged particle placed in a magnetic field generated by the vector potential $\vec{A}(\rho, \phi, z)=\frac{c k}{e \rho} \hat{e}_{z}$, where $k$ is a constant characterizing the field strength, $c$ is the speed of light, $e$ is the charge of the particle, and $\rho$ is the radial variable in cylindrical coordinates. The resulting field has cylindrical symmetry, its intensity decreases with the distance to the symmetry axis and its field lines are parallel to the $x-y$ plane. Making use of the basic ideas to solve the shape invariant potentials through the 1-SUSY QM technique, we will work out the same problem in

Section 3 in the relativistic regime by solving the associated Dirac equation. In the last section we will present our conclusions.

\section{Nonrelativistic quantum approach}

The classical Hamiltonian of a particle with charge $e$ and mass $m$ in a magnetic field generated by the vector potential $\vec{A}(\rho, \phi, z)=\frac{c k}{e \rho} \hat{e}_{z}$ is given by

$$
H_{\mathrm{cl}}=\frac{P_{x}^{2}}{2 m}+\frac{P_{y}^{2}}{2 m}+\frac{1}{2 m}\left(P_{z}-\frac{k}{\rho}\right)^{2} \text {. }
$$

The corresponding magnetic field $\vec{B}=\nabla \times \vec{A}$ could be produced in a coaxial transmission line, with the inner and outer conductors carrying currents $I_{a}$ and $-I_{a} \tau /\left(r_{o}+\tau\right)$ respectively, where $r_{o}$ is the minor radius of the outer conductor and $\tau$ is its thickness. If the current density in the second conductor is $\|\vec{J}\|=r_{o} I_{a} /\left(2 \pi \rho^{3}\right)$ then such a magnetic field will be generated in the material $[9,19]$.

In order to address the quantum treatment the classical observables have to be promoted to the corresponding quantum operators. The quantum Hamiltonian is then

$$
H=-\frac{\hbar^{2}}{2 m} \nabla^{2}-\frac{k}{m \rho}\left(-i \hbar \frac{\partial}{\partial z}\right)+\frac{k^{2}}{2 m \rho^{2}},
$$

where $\nabla^{2}$ is the Laplacian operator.

It can be seen that the Hamiltonian commutes with the operators of partial derivative with respect to $z$ and $\phi$,

$$
\left[H, \frac{\partial}{\partial z}\right]=\left[H, \frac{\partial}{\partial \phi}\right]=0 .
$$




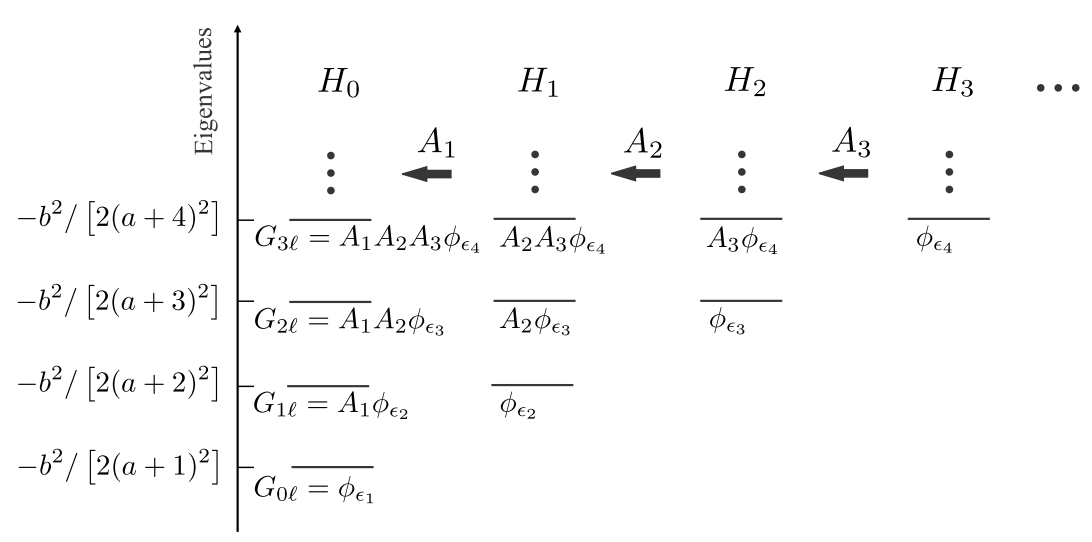

Figure 1. A hierarchy of Hamiltonians built up departing from $H_{0}$. If we know the ground state of each Hamiltonian and the intertwining operators, we can know the bound states of all Hamiltonians. Note that the eigenvalues are not indeed equidistant.

This suggests us the following ansatz for the solutions of the stationary Schrödinger equation

$$
\psi(\rho, \phi, z)=e^{i\left(p_{z} z+\ell \phi\right) / \hbar} \rho^{-1 / 2} G(\rho),
$$

where $p_{z}$ and $\ell$ are respectively the eigenvalues of the momentum operator along $z, P_{z}=$ $-i \hbar \partial / \partial z$, and the $z$ component of the angular momentum, $L_{z}=-i \hbar \partial / \partial \phi$.

Through this ansatz we can separate variables for the stationary Schrödinger equation, $H \psi=$ $E \psi$, leading us to the differential equation for $G(\rho)$

$$
\left[-\frac{1}{2} \frac{\mathrm{d}^{2}}{\mathrm{~d} \rho^{2}}+\frac{(\lambda / \hbar)^{2}-1 / 4}{2 \rho^{2}}-\frac{p_{z} k}{\hbar^{2} \rho}\right] G(\rho)=\frac{m}{\hbar^{2}}\left(E-\frac{p_{z}^{2}}{2 m}\right) G(\rho),
$$

where $\lambda^{2}=\ell^{2}+k^{2}$. To simplify notation we can express the previous equation as

$$
\left[-\frac{1}{2} \frac{\mathrm{d}^{2}}{\mathrm{~d} \rho^{2}}+\frac{a(a+1)}{2 \rho^{2}}-\frac{b}{\rho}\right] G(\rho)=d G(\rho),
$$

with

$$
a(a+1)=\frac{\lambda^{2}}{\hbar^{2}}-\frac{1}{4}, \quad b=\frac{p_{z} k}{\hbar^{2}}, \quad d=\frac{m E}{\hbar^{2}}-\frac{p_{z}^{2}}{2 \hbar^{2}} .
$$

In this work we restrict ourselves to the case $p_{z} k>0$, which is the one with bound states. Equation (6) can be identified as the radial equation of the hydrogen atom. To solve this equation we propose the existence of a family of operators $A_{n}^{\dagger}$ that intertwine the Hamiltonians $H_{n}$ and $H_{n+1}$ in the way

$$
H_{n+1} A_{n+1}^{\dagger}=A_{n+1}^{\dagger} H_{n},
$$

where

$$
H_{n}=-\frac{1}{2} \frac{\mathrm{d}^{2}}{\mathrm{~d} \rho^{2}}+V_{n}(\rho)=-\frac{1}{2} \frac{\mathrm{d}^{2}}{\mathrm{~d} \rho^{2}}+\frac{(a+n)(a+n+1)}{2 \rho^{2}}-\frac{b}{\rho}
$$

and

$$
A_{n}^{\dagger}=\frac{1}{\sqrt{2}}\left(-\frac{\mathrm{d}}{\mathrm{d} \rho}+W_{n}(\rho)\right)
$$


From equations (2) we have

$$
W_{n}(\rho)=\frac{a+n}{\rho}-\frac{b}{a+n}, \quad \epsilon_{n}=-\frac{b^{2}}{2(a+n)^{2}} .
$$

Looking for the function annihilated by $A_{n}^{\dagger}$, which due to equation (3) is an eigenfunction of $H_{n-1}$ with eigenvalue $\epsilon_{n}$,

$$
A_{n}^{\dagger} \phi_{\epsilon_{n}}=0 \Rightarrow \phi_{\epsilon_{n}}=\rho^{a+n} e^{-\frac{b}{a+n} \rho}
$$

it turns out that the ground state of $H_{n}$ is given by

$$
\phi_{\epsilon_{n+1}}(\rho)=\rho^{a+n+1} e^{-\frac{b}{a+n+1} \rho} .
$$

As expected, one can verify that

$$
W_{n+1}(\rho)=\phi_{\epsilon_{n+1}}^{\prime}(\rho) / \phi_{\epsilon_{n+1}}(\rho) .
$$

It is enough to know the ground state and its eigenvalue for any Hamiltonian of the hierarchy in order to find the complete solution of $H_{0}$ (see Fig. 1). The spectrum is given by

$$
\operatorname{Sp}\left[H_{0}\right]=\left\{-\frac{b^{2}}{2(a+n+1)^{2}}, n=0,1,2, \ldots\right\},
$$

and its eigenfunctions by

$$
G_{0 \ell}=\phi_{\epsilon_{1}}, \quad G_{1 \ell}=A_{1} \phi_{\epsilon_{2}}, \quad G_{2 \ell}=A_{1} A_{2} \phi_{\epsilon_{3}}, \quad G_{3 \ell}=A_{1} A_{2} A_{3} \phi_{\epsilon_{4}}, \quad \ldots
$$

where the index $n$ indicates the energy level of $H_{0}$ and the index $\ell$ reminds us that the radial Hamiltonian depends on the angular momentum. In Fig. 2 the first three eigenfunctions of $H_{0}$ can be seen (black continuous, dashed and dotted lines) placed at its corresponding energy level and the potential $V_{0}(\rho)$ is as well drawn (gray line).

Returning to the original problem, i.e. the eigenvalue equation for the operator of equation (4), we have that the spectrum is given by

$$
\operatorname{Sp}[H]=\left\{\frac{p_{z}^{2}}{2 m}\left[1-\frac{k^{2}}{\hbar^{2}(\lambda / \hbar+n+1 / 2)^{2}}\right], n=0,1,2, \ldots\right\},
$$

and its eigenfunctions by

$$
\psi_{n \ell p_{z}}(\rho, \phi, z)=C_{n \ell p_{z}} e^{i\left(p_{z} z+\ell \phi\right) / \hbar} \rho^{-1 / 2} G_{n \ell}(\rho),
$$

where $C_{n \ell p_{z}}$ is a normalization constant (there is not sum convention).

\section{Relativistic quantum approach}

The stationary Dirac equation of a free particle with mass $m$ and spin $1 / 2$ is

$$
\mathbf{H}_{D} \Psi=\left[c \vec{\alpha} \cdot \overrightarrow{\mathbf{P}}+\beta m c^{2}\right] \Psi=E \Psi,
$$

where $\overrightarrow{\mathbf{P}}$ is the momentum operator, $\Psi$ is a four-component spinor and $\alpha_{i}$ and $\beta$ are $4 \times 4$ matrices given by

$$
\alpha_{i}=\left(\begin{array}{cc}
0 & \sigma_{i} \\
\sigma_{i} & 0
\end{array}\right), \quad \beta=\left(\begin{array}{cc}
\sigma_{0} & 0 \\
0 & -\sigma_{0}
\end{array}\right),
$$




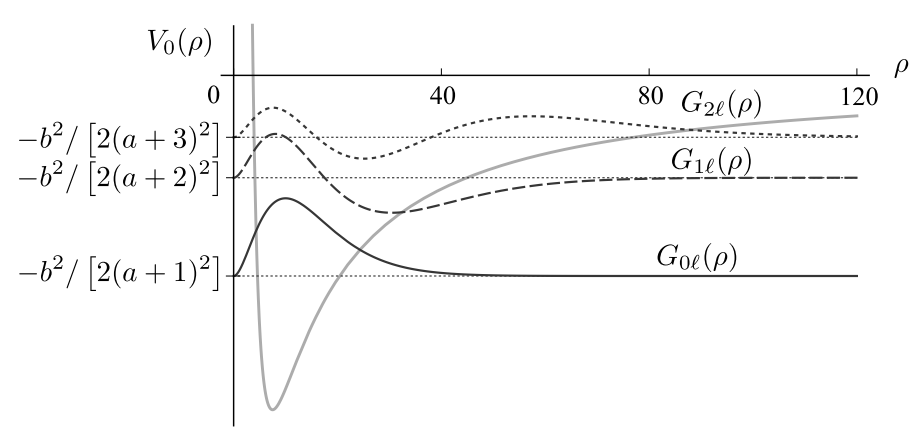

Figure 2. The potential $V_{0}(\rho)$ (gray curve) and its first three eigenfunctions, $G_{0 \ell}$ (black continuous line), $G_{1 \ell}$ (dashed line) and $G_{2 \ell}$ (dotted line), with $a=1.5$ and $b=0.5$ in units of $1 / \rho$.

being $\sigma_{0}$ the $2 \times 2$ identity matrix and $\sigma_{i}$ the Pauli matrices. The $4 \times 4$ matrix operators are written in boldface in order to be distinguished from the $2 \times 2$ matrix operators. In our case the interaction with the magnetic field derived from the vector potential $\overrightarrow{\mathbf{A}}=\frac{c k}{e \rho} \hat{e}_{z}$ is described by the minimal coupling rule $\overrightarrow{\mathbf{P}} \rightarrow \overrightarrow{\mathbf{P}}-\frac{e}{c} \overrightarrow{\mathbf{A}}$. In cylindrical coordinates the resulting stationary Dirac equation is

$$
\mathbf{H}_{D} \Psi=\left\{-i \hbar c \mathbf{D}(\phi) \alpha_{1} \frac{\partial}{\partial \rho}-\frac{i \hbar c}{\rho} \mathbf{D}(\phi) \alpha_{2} \frac{\partial}{\partial \phi}-i \hbar c \alpha_{3} \frac{\partial}{\partial z}-\frac{k}{\rho} \alpha_{3}+\beta m c^{2}\right\} \Psi=E \Psi,
$$

where $\mathbf{D}(\phi)=\operatorname{Diag}\left[e^{-i \phi}, e^{i \phi}, e^{-i \phi}, e^{i \phi}\right]$ is a diagonal matrix. This interacting Hamiltonian commutes with the momentum operator, and with the total angular momentum in the $z$-direction,

$$
\mathbf{P}_{z}=-i \hbar \partial_{z} \mathbb{1}, \quad \mathbf{J}_{z}=-i \hbar \partial_{\phi} \mathbb{1}+\frac{\hbar}{2} \Sigma_{3}, \quad \Sigma_{i}=\left(\begin{array}{cc}
\sigma_{i} & 0 \\
0 & \sigma_{i}
\end{array}\right) .
$$

Then we will look for a solution to equation (9) that is also an eigenfunction of these two operators with corresponding eigenvalues $p_{z}$ and $\ell$ respectively (see equation (5)), having the form

$$
\Psi(\rho, \phi, z)=e^{i p_{z} z / \hbar} e^{i\left(\ell \mathbb{1}-\Sigma_{3} / 2\right) \phi / \hbar} \rho^{-1 / 2} G_{D}(\rho) .
$$

The equation that the radial function $G_{D}(\rho)$ must fulfill is

$$
\left[-i \alpha_{1} \frac{\mathrm{d}}{\mathrm{d} \rho}+\frac{\ell}{\hbar \rho} \alpha_{2}-\left(\frac{k}{\hbar \rho}-\frac{p_{z}}{\hbar}\right) \alpha_{3}+\frac{m c}{\hbar} \beta\right] G_{D}(\rho)=\frac{E}{c \hbar} G_{D}(\rho) .
$$

The operator between brackets is an effective Hamiltonian that will be called $\mathbf{H}_{\rho}$. It is useful to perform an unitary transformation in order to leave all the dependence of $\rho$ on a single matrix,

$$
\mathbf{H}_{0}=\mathbf{U}_{1}^{\dagger} \mathbf{H}_{\rho} \mathbf{U}_{1}, \quad \mathbf{U}_{1}=e^{-i \theta \Sigma_{1} / 2}=\cos (\theta / 2) \mathbb{1}-i \sin (\theta / 2) \Sigma_{1},
$$

with $\tan \theta=-k / \ell$. In the rotated frame the Hamiltonian is

$$
\mathbf{H}_{0}=-i \alpha_{1} \frac{\mathrm{d}}{\mathrm{d} \rho}+\left(\frac{\lambda}{\hbar \rho}-\frac{p_{z} k}{\hbar \lambda}\right) \alpha_{2}+\frac{p_{z} \ell}{\hbar \lambda} \alpha_{3}+\frac{m c}{\hbar} \beta
$$

where in this context once again $\lambda^{2}=\ell^{2}+k^{2}$. This Hamiltonian has a special structure that can be better appreciated if we write it as follows

$$
\mathbf{H}_{0}=\left(\begin{array}{cc}
(m c / \hbar) \sigma_{0} & h_{0} \\
h_{0} & -(m c / \hbar) \sigma_{0}
\end{array}\right), \quad h_{0}=-i \sigma_{1} \frac{\mathrm{d}}{\mathrm{d} \rho}+\left(\frac{\lambda}{\hbar \rho}-\frac{p_{z} k}{\hbar \lambda}\right) \sigma_{2}+\frac{p_{z} \ell}{\hbar \lambda} \sigma_{3},
$$


being $h_{0}$ a $2 \times 2$ matrix operator. In order to simplify notation we will write

$$
h_{0}=-i \sigma_{1} \frac{\mathrm{d}}{\mathrm{d} \rho}+\left(\frac{a}{\rho}-\frac{b_{0}}{a}\right) \sigma_{2}+d_{0} \sigma_{3}=-i \sigma_{1} \frac{\mathrm{d}}{\mathrm{d} \rho}+v_{0}\left(a, b_{0}, d_{0} ; \rho\right),
$$

with

$$
a=\lambda / \hbar, \quad b_{0}=p_{z} k / \hbar^{2}, \quad d_{0}=p_{z} \ell / \hbar \lambda .
$$

To solve the eigenvalue equation for $\mathbf{H}_{0}$ with a method similar to that used in the nonrelativistic approach, we propose the intertwining relationship

$$
\mathbf{H}_{n+1} \mathbf{A}_{n+1}^{\dagger}=\mathbf{A}_{n+1}^{\dagger} \mathbf{H}_{n},
$$

with the intertwining operators $\mathbf{A}_{n+1}^{\dagger}$ and the sequence of Hamiltonians $\mathbf{H}_{n+1}$ having the form

$$
\mathbf{A}_{n+1}^{\dagger}=\left(\begin{array}{cc}
B_{n+1}^{\dagger} & 0 \\
0 & B_{n+1}^{\dagger}
\end{array}\right), \quad \mathbf{H}_{n}=\left(\begin{array}{cc}
(m c / \hbar) \sigma_{0} & h_{n} \\
h_{n} & -(m c / \hbar) \sigma_{0}
\end{array}\right),
$$

where $h_{n}\left(a_{n}, b_{n}, d_{n} ; \rho\right)=h_{0}\left(a+n, b_{n}, d_{n} ; \rho\right)$ with parameters $b_{n}$ and $d_{n}$ to be determined and $B_{n+1}^{\dagger}$ is a $2 \times 2$ operator that intertwines $h_{n+1}$ with $h_{n}$. The structure of $\mathbf{A}_{n+1}^{\dagger}$ above proposed is the simplest choice. A more general form of the intertwining operators will be presented elsewhere. Similar $2 \times 2$ intertwining operators were considered in [10].

In the same way as in the nonrelativistic quantum case (see equation (1)), the first order intertwining operator has the following structure

$$
\mathbf{A}_{n+1}^{\dagger}=-\mathbb{1} \frac{\mathrm{d}}{\mathrm{d} \rho}+\mathbf{W}_{n+1}^{\dagger}(\rho)
$$

where $\mathbf{W}_{n+1}^{\dagger}(\rho)$ is a variable matrix. In the same way $B_{n+1}^{\dagger}$ reads

$$
B_{n+1}^{\dagger}=-\sigma_{0} \frac{\mathrm{d}}{\mathrm{d} \rho}+F_{n+1}(\rho)
$$

with $F_{n+1}(\rho)$ a $2 \times 2$ matrix.

Solving the intertwining relation of equation (11) we find

$$
\begin{aligned}
b_{n}=b_{0}= & b, \quad d_{n}^{2}=d_{0}^{2}+\frac{n(2 a+n) b^{2}}{a^{2}(a+n)^{2}}, \\
B_{n+1}^{\dagger}= & -\sigma_{0} \frac{\mathrm{d}}{\mathrm{d} \rho}+\frac{2(a+n)+1}{2}\left[\frac{1}{\rho}-\frac{b}{(a+n)(a+n+1)}\right] \sigma_{0}-\frac{d_{n+1}-d_{n}}{2}\left(i \sigma_{1}-\sigma_{2}\right) \\
& -\frac{1}{2}\left[\frac{1}{\rho}+\frac{b}{(a+n)(a+n+1)}\right] \sigma_{3} .
\end{aligned}
$$

Then the intertwining operators $\mathbf{A}_{n+1}^{\dagger}$ are directly obtained by substitution in equation (12).

Now, we look for the vector wavefunctions annihilated by $\mathbf{A}_{n+1}^{\dagger}$. Taking advantage of the block diagonal structure of this operator, first we find the two-component functions annihilated by $B_{n+1}^{\dagger}$. These are given by the following two independent vector functions

$$
\begin{aligned}
& \chi_{n}=\left(\begin{array}{l}
1 \\
0
\end{array}\right) \rho^{a+n} e^{-b \rho /(a+n)}, \\
& \xi_{n}=\left(i \frac{(a+n)^{2}(a+n+1)^{2}\left(d_{n+1}-d_{n}\right)}{b^{2}}\left(1-\frac{b}{(a+n)(a+n+1)} \rho\right)\right) \rho^{a+n} e^{-b \rho /(a+n+1)} .
\end{aligned}
$$


We can built four-component functions annihilated by $\mathbf{A}_{n+1}^{\dagger}$ such that at the same time they are also eigenvectors of $\mathbf{H}_{n}$. We find four types of such vectors and the corresponding eigenvalues:

$$
\begin{aligned}
& \phi_{a n}=\left(\begin{array}{c}
\chi_{n} \\
\frac{d_{n}}{\sqrt{(m c / \hbar)^{2}+d_{n}^{2}}+m c / \hbar} \chi_{n}
\end{array}\right), \quad \sqrt{(m c / \hbar)^{2}+d_{n}^{2}} ; \\
& \phi_{b n}=\left(\begin{array}{c}
\chi_{n} \\
-\frac{d_{n}}{\sqrt{(m c / \hbar)^{2}+d_{n}^{2}}-m c / \hbar} \chi_{n}
\end{array}\right), \quad-\sqrt{(m c / \hbar)^{2}+d_{n}^{2}} ; \\
& \phi_{c n}=\left(-\frac{\xi_{n}}{\sqrt{(m c / \hbar)^{2}+d_{n+1}^{2}}+m c / \hbar} \xi_{n}\right), \quad \sqrt{(m c / \hbar)^{2}+d_{n+1}^{2}} ; \\
& \phi_{d n}=\left(\frac{\xi_{n}}{\sqrt{(m c / \hbar)^{2}+d_{n+1}^{2}}-m c / \hbar} \xi_{n}\right), \quad-\sqrt{(m c / \hbar)^{2}+d_{n+1}^{2}} \text {. }
\end{aligned}
$$

We will briefly comment here on some properties of these results, a more complete discussion will be given elsewhere.

- Superpotential. Consider now the matrix $\Xi_{n+1}(\rho)$ which is constructed by placing the vectors $\phi_{\nu n}$ in its columns, it can be verified, in analogy with equation (7), that

$$
\mathbf{W}_{n+1}^{\dagger}(\rho)=-\Xi_{n+1}^{\prime}(\rho) \Xi_{n+1}^{-1}(\rho) .
$$

- Spectrum. From the intertwining relationship, equation (11), it is shown that the spectrum of $\mathbf{H}_{0}$ is given by

$$
\operatorname{Sp}\left[\mathbf{H}_{0}\right]= \pm \sqrt{\left(\frac{m c}{\hbar}\right)^{2}+d_{0}^{2}+\frac{n(2 a+n) b^{2}}{a^{2}(a+n)^{2}}}, \quad n=0,1, \ldots
$$

- Eigenfunctions. The eigenfunctions of the initial Hamiltonian are computed in the usual way. We have four types of eigenfunctions:

$$
\begin{array}{llll}
\Phi_{a 0 \ell}=\phi_{a 0}, & \Phi_{a 1 \ell}=\mathbf{A}_{1} \phi_{a 1}, & \Phi_{a 2 \ell}=\mathbf{A}_{1} \mathbf{A}_{2} \phi_{a 2}, & \ldots, \\
\Phi_{b 0 \ell}=\phi_{b 0}, & \Phi_{b 1 \ell}=\mathbf{A}_{1} \phi_{b 1}, & \Phi_{b 2 \ell}=\mathbf{A}_{1} \mathbf{A}_{2} \phi_{b 2}, & \ldots, \\
\Phi_{c 0 \ell}=\phi_{c 0}, & \Phi_{c 1 \ell}=\mathbf{A}_{1} \phi_{c 1}, & \Phi_{c 2 \ell}=\mathbf{A}_{1} \mathbf{A}_{2} \phi_{c 2}, & \ldots, \\
\Phi_{d 0 \ell}=\phi_{d 0}, & \Phi_{d 1 \ell}=\mathbf{A}_{1} \phi_{d 1}, & \Phi_{d 2 \ell}=\mathbf{A}_{1} \mathbf{A}_{2} \phi_{d 2}, & \ldots,
\end{array}
$$

where we add the subindex $\ell$ to remind that the Hamiltonian $\mathbf{H}_{0}$ depends on $\ell$.

Then the eigenvectors of the Dirac equation, equation (8), for our system are

$$
\Psi_{\nu n \ell p_{z}}(\rho, \phi, z)=C_{\nu n \ell p_{z}} e^{i p_{z} z / \hbar} e^{i\left(\ell \mathbb{1}-\frac{1}{2} \Sigma_{3}\right) \phi / \hbar} \mathbf{U}_{1} \rho^{-1 / 2} \Phi_{\nu n \ell}(\rho),
$$

where $\mathbf{U}_{1}$ is given by equation (10), $\nu=a, b, c, d, n=0,1,2, \ldots$ and $C_{\nu n \ell p_{z}}$ are normalization constants (there is not sum convection). The spectrum of $\mathbf{H}_{D}$ is $c \hbar$ times the one of $\mathbf{H}_{0}$, thus

$$
\operatorname{Sp}\left[\mathbf{H}_{D}\right]= \pm m c^{2} \sqrt{1+\frac{p_{z}^{2}}{m^{2} c^{2}}-\frac{p_{z}^{2} k^{2}}{\hbar^{2} m^{2} c^{2}(\lambda / \hbar+n)^{2}}}, \quad n=0,1,2, \ldots
$$




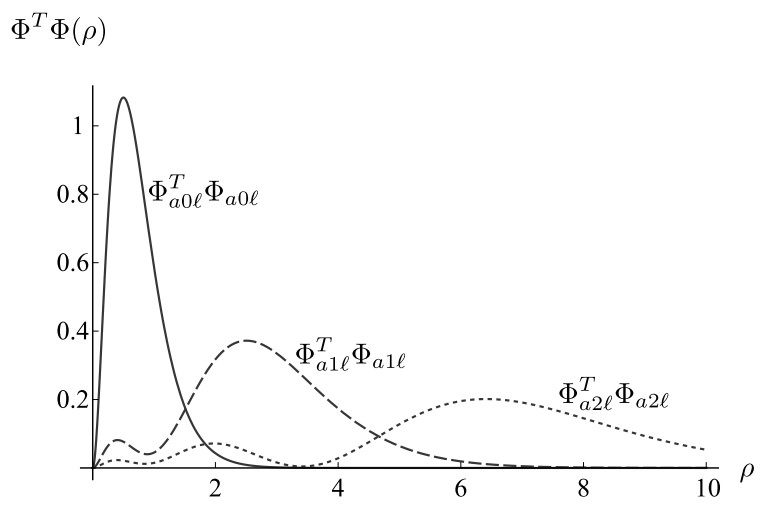

(a)

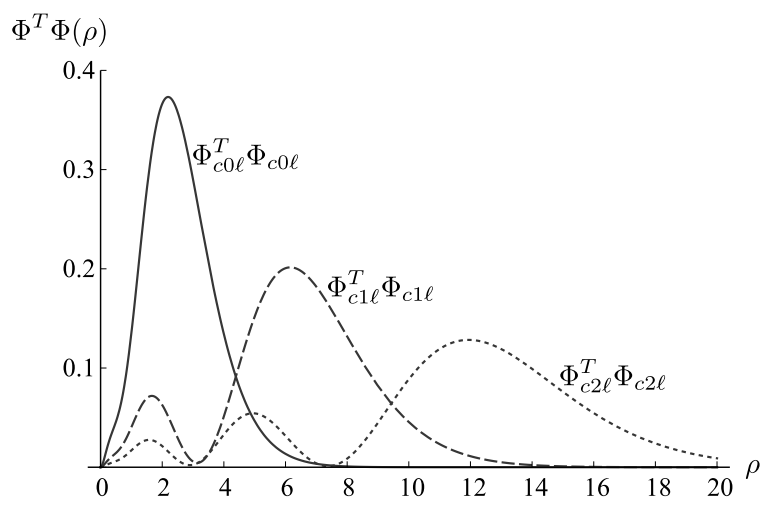

(b)

Figure 3. Probability densities for six eigenvectors of $\mathbf{H}_{0}$ : in $(a)$ the first three of the family $\Phi_{\text {an } \ell}$, and in $(b)$ the first three of the family $\Phi_{c n \ell}$, the parameters used were $a=1 ; b=2$ and $d_{0}=1$ with units of $1 / \rho ; m=0.1$ and $c=\hbar=1$.

In Fig. 3 we can see the probability densities of six eigenvectors of $\mathbf{H}_{0}$. In $(a)$ we have the first three with subindex $a$, and in $(b)$ the corresponding but with subindex $c$. Note that $\Phi_{a 1 \ell}$ and $\Phi_{c 0 \ell}$ have the same eigenvalue but the behavior of the probability density is quite different, the same happens with $\Phi_{a 2 \ell}$ and $\Phi_{c 1 \ell}$, and so on. This degeneracy, which does not appear in the nonrelativistic approach, is due to the spin degree of freedom and will be analyzed in detail elsewhere.

\section{Conclusions}

In this work we have adapted the intertwining technique to solve exactly the Dirac equation associated to a charged particle of spin $1 / 2$ immersed in a magnetic field with cylindrical symmetry generated by the vector potential $\vec{A}=\frac{c k}{e \rho} \hat{e}_{z}$. We first addressed the problem in the nonrelativistic regime, i.e., the Schrödinger equation through the standard intertwining technique. Afterwards we set up the corresponding Dirac equation and we proposed, as in the nonrelativistic approach, a hierarchy of shape invariant Hamiltonians intertwined by some operators to be determined. These operators afterwards were found and using them the ground states of each Hamiltonian were built. Applying these operators onto the ground states all the bound states were obtained as well as their respective eigenvalues of the original Dirac equation. As far as we know these solutions have not been reported before. The analogies between the method for solving the Dirac equation and the standard intertwining technique for the Schrödinger equation were recurrently employed throughout the entire procedure.

\section{Acknowledgments}

We acknowledge financial support from Ministerio de Ciencia e Innovación (MICINN) of Spain, projects MTM2009-10751, and FIS2009-09002. ACA acknowledges to Conacyt a PhD grant and the kind hospitality at University of Valladolid. DJFC acknowledges the financial support of Conacyt, project 152574 .

\section{References}

[1] Bagrov V.G., Samsonov B.F., Darboux transformation and elementary exact solutions of the Schrödinger equation, Pramana J. Phys. 49 (1997), 563-580.

[2] Castaños O., Frank A., López R., Urrutia L.F., Soluble extensions of the Dirac oscillator with exact and broken supersymmetry, Phys. Rev. D 43 (1991), 544-547. 
[3] Contreras-Astorga A., Fernández C. D.J., Supersymmetric partners of the trigonometric Pöschl-Teller potentials, J. Phys. A: Math. Theor. 41 (2008), 475303, 18 pages, arXiv:0809.2760.

[4] Cooper F., Khare A., Sukhatme U., Supersymmetry and quantum mechanics, Phys. Rep. 251 (1995), 267385, hep-th/9405029.

[5] de Lima Rodrigues R., Generalized ladder operators for the Dirac-Coulomb problem via SUSY QM, Phys. Lett. A 326 (2004), 42-46, hep-th/0311091.

[6] Debergh N., Pecheritsin A.A., Samsonov B.F., Van den Bossche B., Darboux transformations of the onedimensional stationary Dirac equation, J. Phys. A: Math. Gen. 35 (2002), 3279-3287, quant-ph/0111163.

[7] Fernández C. D.J., New hydrogen-like potentials, Lett. Math. Phys. 8 (1984), 337-343.

[8] Fernández C. D.J., Fernández-García N., Higher-order supersymmetric quantum mechanics, AIP Conf. Proc. 744 (2005), 236-273, quant-ph/0502098.

[9] Griffiths D.J., Introduction to electrodynamics, 3rd ed., Addison-Wesley, 1999.

[10] Ioffe M.V., Kuru Ş., Negro J., Nieto L.M., SUSY approach to Pauli Hamiltonians with an axial symmetry, J. Phys. A: Math. Gen. 39 (2006), 6987-7001, hep-th/0603005.

[11] Jakubsky V., Nieto L.M., Plyushchay M.S., Klein tunneling in carbon nanostructures: a free-particle dynamics in disguise, Phys. Rev. D 83 (2011), 047702, 4 pages, arXiv:1010.0569.

[12] Jakubsky V., Plyushchay M.S., Supersymmetric twisting of carbon nanotubes, Phys. Rev. D 85 (2012), 045035, 10 pages, arXiv:1111.3776.

[13] Junker G., Roy P., Conditionally exactly solvable potentials: a supersymmetric construction method, Ann. Physics 270 (1998), 155-177, quant-ph/9803024.

[14] Khare A., Supersymmetry in quantum mechanics, AIP Conf. Proc. 744 (2005), 133-165, math-ph/0409003.

[15] Mielnik B., Factorization method and new potentials with the oscillator spectrum, J. Math. Phys. 25 (1984), $3387-3389$.

[16] Nieto L.M., Pecheritsin A.A., Samsonov B.F., Intertwining technique for the one-dimensional stationary Dirac equation, Ann. Physics 305 (2003), 151-189, quant-ph/0307152.

[17] Pozdeeva E., Schulze-Halberg A., Darboux transformations for a generalized Dirac equation in two dimensions, J. Math. Phys. 51 (2010), 113501, 15 pages, arXiv:0904.0992.

[18] Rosas-Ortiz J.O., New families of isospectral hydrogen-like potentials, J. Phys. A: Math. Gen. 31 (1998), L507-L513, quant-ph/9803029.

[19] Sadiku M.N.O., Elements of electromagnetics, 5th ed., The Oxford Series in Electrical and Computer Engineering, Oxford University Press, New York, 2009.

[20] Sukumar C.V., Supersymmetry and the Dirac equation for a central Coulomb field, J. Phys. A: Math. Gen. 18 (1985), L697-L701.

[21] Sukumar C.V., Supersymmetry, factorisation of the Schrödinger equation and a Hamiltonian hierarchy, J. Phys. A: Math. Gen. 18 (1985), L57-L61. 